\title{
Rigorous results on the threshold network model
}

\author{
Norio Konno ${ }^{1}$, Naoki Masuda ${ }^{2}$, Rahul Roy $^{3}$ and Anish Sarkar ${ }^{3}$ \\ ${ }^{1}$ Faculty of Engineering, Yokohama National University, 79-5, Tokiwadai, Hodogaya, \\ Yokohama, 240-8501, Japan \\ ${ }^{2}$ Laboratory for Mathematical Neuroscience, RIKEN Brain Science Institute, 2-1, \\ Hirosawa, Wako, Saitama, 351-0198, Japan \\ ${ }^{3}$ Indian Statistical Institute, 7 SJS Sansanwal Marg, New Delhi 110016, India
}

\begin{abstract}
We analyze the threshold network model in which a pair of vertices with random weights are connected by an edge when the summation of the weights exceeds a threshold. We prove some convergence theorems and central limit theorems on the vertex degree, degree correlation, and the number of prescribed subgraphs. We also generalize some results in the spatially extended cases.

Keywords: complex network, threshold graph, network motif, central limit theorem, Ustatistics
\end{abstract}

PACS numbers: 89.75.Hc, 89.75.Da, 89.75.Fb 


\section{Introduction}

Recently, real large-scale network data have been analyzed in various fields, and the corresponding random graphs have been studied. Many of these graphs exhibit the power-law form of the degree distribution, with the power-law exponent typically between 2 and 3 . In view of this, physicists have proposed stochastic algorithms for generating growing networks. The underlying assumption of these growing networks is that a vertex with a certain fixed number of edges is added to the graph one by one at each discrete time step. To obtain a power law, the growth mechanism is usually supplied by so-called preferential attachment, which stipulates that each newly introduced edge is more likely to be connected to a vertex (which already exists in the graph) that has a larger degree. For a review of the studies on such models see [1, 2].

However, not all real networks are growing; algorithms for generating nongrowing realistic graphs could be more appropriate for real situations in which the number of vertices does not change rapidly. In this regard, a type of random graph in which each of $n$ vertices is assigned a random variable (weight) was proposed and its mean behavior has been analyzed [3, 4, 5, 6]. Interestingly, the power-law degree distribution can emerge even with a weight distribution that is not equipped with a power-law. For example, mean-field analysis suggests a power-law degree distribution with scaling exponent -2 when the vertex weights are independent and identically distributed (i.i.d.) random variables obeying the exponential distribution.

Our first study is regarding such graphs. Formally, our model consists of $n$ vertices labeled $1, \ldots, n$ and an i.i.d. sequence of random variables $X_{1}, \ldots, X_{n}$ with $F$ denoting their common distribution function. We associate the random variable $X_{i}$ with the vertex labeled $i$. Given a fixed threshold value $\theta>0$, we connect the vertices $i$ and $j$ by an edge $\langle i, j\rangle$ if and only if $X_{i}+X_{j}>\theta$ and $i \neq j$. Let $G_{\theta}$ be the random graph thus produced. A simple coupling argument shows that $\mathbb{P}\left\{\langle i, j\rangle \in G_{\theta^{\prime}}\right\} \leq \mathbb{P}\left\{\langle i, j\rangle \in G_{\theta}\right\}$ whenever $\theta^{\prime} \geq \theta$.

Let $D_{n}(i):=\#\left\{j:\langle i, j\rangle \in G_{\theta}\right\}$, i.e. the degree of the vertex $i$ in the graph $G_{\theta}$. Note $\left\{D_{n}(i): i \geq 1\right\}$ are identical in distribution and let $D_{n}$ denote a random variable with this common distribution. The distribution $D_{n}$ can be obtained as follows: given $(n+1)$ vertices, conditioned on the event $X_{1}=x$, vertices $j \in\{2, \ldots, n+1\}$, will connect to the vertex 1 if and only if $X_{j}>\theta-x$. 
Therefore, for $0 \leq k \leq n$, we have

$$
\mathbb{P}\left(D_{n+1}=k\right)=\int_{-\infty}^{\infty}\left(\begin{array}{l}
n \\
k
\end{array}\right)[1-F(\theta-x)]^{k}[F(\theta-x)]^{n-k} F(d x) .
$$

Here $F(d x)$ represents the probability measure on the real line representing the law of $X_{1}$. Equation (11) allows us to obtain the following asymptotic result:

Theorem 1 As $n \rightarrow \infty$,

$$
\frac{D_{n}}{n} \Longrightarrow 1-F\left(\theta-X_{1}\right) .
$$

Here $\Longrightarrow$ stands for convergence in distribution.

In data-analysis context, we are often concerned with the degree correlation between a pair of vertices [1, 2]. Here we discuss the asymptotic properties of the graph. Our first result is

Theorem $2 \frac{D_{n}(1)}{n}$ and $\frac{D_{n}(2)}{n}$ are asymptotically independent.

This asymptotic independence breaks under the condition that the vertices 1 and 2 are connected. However, we need to be careful about this statement, in the sense that we will need some conditions on the distribution function $F$ of the weight of a vertex. In particular we assume that

Assumption 1: There exists $u$ and $v$ in the support of the distribution function $F$ (i.e. for any $\epsilon>0, F(u+\epsilon)>F(u-\epsilon)$ and $F(v+\epsilon)>F(v-\epsilon)$ ) such that $u<\theta / 2<v$ and $u+v>\theta$.

Theorem 3 Given that the vertices 1 and 2 are connected, under the above assumption $\frac{D_{n}(1)}{n}$ and $\frac{D_{n}(2)}{n}$ are not asymptotically independent.

The importance of the assumption can be understood by looking at the situation when the assumption does not hold. In that case, the vertices can be classified into two groups: those which have weights $\theta / 2$ or less and those which have weights more than $\theta / 2$. In the random graph constructed, each of the vertices of the former group remain isolated, whereas the vertices of the latter group form a complete graph among themselves. Also, by the strong law of large numbers, the probability that a vertex belongs to the former group converges to $F(\theta / 2)$ and the probability that a vertex belongs to the latter group converges to $1-F(\theta / 2)$. Moreover, given two vertices ( 1 and 2 (say)) are connected, they must belong to the latter group and then we have

$$
\begin{aligned}
& \mathbb{P}\left[\lim _{n \rightarrow \infty} \frac{D_{n}(1)}{n}=1-F(\theta / 2), \lim _{n \rightarrow \infty} \frac{D_{n}(2)}{n}=1-F(\theta / 2) \mid \text { vertices } 1 \text { and } 2 \text { are connected }\right] \\
& =\mathbb{P}\left[\lim _{n \rightarrow \infty} \frac{D_{n}(1)}{n}=1-F(\theta / 2) \mid \text { vertices } 1 \text { and } 2 \text { are connected }\right]=1
\end{aligned}
$$


Thus we obtain conditional asymptotic independence.

A remarkable characteristic of real graphs is the clustering property 1, 2, The clustering property means an abundance of connected triangles in the random graph. The threshold model exhibits such a clustering property as shown exactly in [5, 6].

To formalize this, let $h: \mathbb{R}^{3} \rightarrow \mathbb{R}$ be given by

$$
h\left(x_{1}, x_{2}, x_{3}\right):=1_{\left\{x_{1}+x_{2}>\theta, x_{2}+x_{3}>\theta, x_{3}+x_{1}>\theta\right\}} .
$$

Also let

$$
\begin{aligned}
T_{n}:=\#\{(i, j, k): 1 \leq i<j<k \leq n, \\
\left.X_{i}+X_{j}>\theta, X_{j}+X_{k}>\theta, X_{k}+X_{i}>\theta\right\}, \\
F_{3}(\theta):=\mathbb{E}\left[h\left(X_{1}, X_{2}, X_{3}\right)\right] \\
=\int_{\mathbb{R}} \int_{\mathbb{R}} \int_{\mathbb{R}} F\left(d x_{1}\right) F\left(d x_{2}\right) F\left(d x_{3}\right) h\left(x_{1}, x_{2}, x_{3}\right) \\
=\mathbb{P}\left(X_{1}+X_{2}>\theta, X_{2}+X_{3}>\theta, X_{3}+X_{1}>\theta\right), \\
\zeta_{1}(F):=\mathbb{E}\left[\left(\int_{\mathbb{R}} \int_{\mathbb{R}} F\left(d x_{2}\right) F\left(d x_{3}\right) h\left(X_{1}, x_{2}, x_{3}\right)\right)^{2}\right]-\left(F_{3}(\theta)\right)^{2}>0 .
\end{aligned}
$$

Note that $T_{n}$ counts all triangles in the graph with $n$ vertices.

The asymptotic results are

Theorem 4 As $n \rightarrow \infty$,

(a) $\frac{T_{n}}{\left(\begin{array}{l}n \\ 3\end{array}\right)} \rightarrow F_{3}(\theta)$ almost surely;

(b) $\sqrt{n}\left[\frac{T_{n}}{\left(\begin{array}{l}n \\ 3\end{array}\right)}-F_{3}(\theta)\right] \Rightarrow \sqrt{3 \zeta_{1}(F)} Z$ where $Z$ is a standard normal random variable.

The method used to show the above results may be generalized easily to obtain a count of not only triangles but subgraphs in $G_{\theta}$ isomorphic to a fixed graph. In data-analysis contexts, a fixed subgraph is called a motif of the graph. Depending on the types of real networks (e.g. Internet, gene networks, neural networks, social networks), there are some sorts of small motifs that appear in an entire graph significantly more than in the random graphs. These motifs are relevant to functional roles such as signal transduction and information processing apposite to each application [8, 9]. Our results, Theorems 8 and 9 in Section 4, obtain limit theorems for the motifs of the threshold model. 
Besides the extension to general graphs, the above theorem may be extended for local triangle counts, i.e., the number of triangles containing a specified vertex. The clustering coefficient, which is a quantity often used to evaluate the degree of clustering property, is defined as the normalized number of locally counted triangles averaged over all the vertices. Our local results below show that the vertex-wise clustering coefficient satisfies the central limit theorem, which validates the use of expectation in physics community.

We fix the vertex 1 and define $T_{n}(1)$ as the number of triangles in the set of vertices $\{1,2, \ldots, n, n+1\}$ of which the vertex 1 is a site. In other words,

$$
T_{n}(1):=\#\left\{2 \leq i<j \leq n+1: h\left(X_{1}, X_{i}, X_{j}\right)=1\right\} .
$$

Theorem 5 As $n \rightarrow \infty$,

$$
\frac{T_{n}(1)}{\left(\begin{array}{l}
n \\
2
\end{array}\right)} \Rightarrow \int_{\mathbb{R}} \int_{\mathbb{R}} F\left(d x_{1}\right) F\left(d x_{2}\right) h\left(X, x_{1}, x_{2}\right)
$$

where $X$ is an independent random variable identical in distribution to $X_{1}$.

Besides the above dimensionless random graph model, a spatial model has been proposed in [7. Consider a homogeneous Poisson point process of intensity $\lambda$ on $\mathbb{R}^{d}$. We insist that the origin, $\mathbf{0}$, is a point of the process. Let $\left\{\mathbf{0}=\xi_{0}, \xi_{1}, \xi_{2}, \ldots\right\}$ be an enumeration of the points of the process. Associated with each point $\xi_{i}$ is a random variable $X_{i}$. We assume that $\left\{X_{0}, X_{1}, X_{2}, \ldots\right\}$ is an i.i.d. sequence of random variables with common distribution function $F$. The random graph $G_{\theta, \beta}$ is obtained by connecting $\xi_{i}$ and $\xi_{j}$ by an edge if and only if $\left(X_{i}+X_{j}\right)>\theta\left|\xi_{i}-\xi_{j}\right|^{\beta}$ where $\theta$ and $\beta$, the parameters of the model, are real numbers.

Define the degree of the origin in a sphere of radius $r$ as

$$
\Delta_{r}:=\#\left\{i \geq 1:\left(X_{0}+X_{i}\right)>\theta\left|\xi_{i}\right|^{\beta} \text { and }\left|\xi_{i}\right| \leq r\right\}
$$

Given a fixed $x \in \mathbb{R}$, let

$$
f(r ; x)=f(|r| ; x):=1-F\left(\theta|r|^{\beta}-x\right) .
$$

Define

$$
C_{r}(x):=\int_{0}^{r} \tilde{r}^{d-1} f(\tilde{r} ; x) d \tilde{r},
$$

and consider the two following cases:

(i) As $r \rightarrow \infty, C_{r}(x) \rightarrow C(x):=\int_{0}^{\infty} \tilde{r}^{d-1} f(\tilde{r}, x) d \tilde{r}<\infty$ for every $x \in \mathbb{R}$. 
(ii) There exists a sequence $\left\{C_{r}\right\}$ and a function $g(x)$ such that $C_{r} \rightarrow \infty$ and $\frac{C_{r}(x)-C_{r}}{\sqrt{C_{r}}} \rightarrow g(x)$ as $r \rightarrow \infty$ for every $x \in \mathbb{R}$.

For the first case, we have

Theorem 6 If $C_{r}(x) \rightarrow C(x)<\infty$ for every $x \in \mathbb{R}$ as $r \rightarrow \infty$, then we have

$$
\Delta_{r} \Rightarrow \Delta
$$

where the characteristic function of the random variable $\Delta$ is given by $\phi_{\Delta}(t)=$ $\int_{\mathbb{R}} F(d x) \exp \left(-\lambda c_{d} C(x)(1-\exp (i t))\right)$ where $c_{d}$ represents the volume of the $(d-1)$-dimensional unit sphere.

Remark The random variable $\Delta$ represents the degree of the origin in the random graph.

For the second case, we have

Theorem 7 Suppose that there exists a sequence $\left\{C_{r}\right\}$ such that $C_{r} \rightarrow \infty$ and $\frac{C_{r}(x)-C_{r}}{\sqrt{C_{r}}} \rightarrow g(x)$ as $r \rightarrow \infty$ for every $x \in \mathbb{R}$. We have

$$
\frac{\Delta_{r}-\lambda c_{d} C_{r}}{\sqrt{\lambda c_{d} C_{r}}} \Rightarrow Z+\sqrt{\lambda c_{d}} g\left(X_{0}\right) \text { as } r \rightarrow \infty
$$

where $c_{d}$ is as defined in Theorem 6 .

We end this section with an example of $F$ satisfying the condition in Theorem [7. Fix $\beta=1$ and $d=2$. Define $F:[0, \infty) \rightarrow[0,1]$ by

$$
F(x):=1-C x^{-\alpha}
$$

where $0<\alpha<2$ and $C>0$, and

$$
C_{r}:=C \theta^{-\alpha} r^{2-\alpha} /(2-\alpha) .
$$

Simple computations can be carried out to verify that the conditions of Theorem 7 are satisfied in this case with $g(x)=0$ for all $x \in \mathbb{R}$. In the next few sections we prove our results.

\section{Degree $D_{n}$ of a vertex}

To prove Theorem 1 observe that, since $(n+1) / n \rightarrow 1$, it is enough to show that $D_{n+1} / n$ converges weakly to the required random variable. For $\xi \in \mathbb{R}$, we 
have from equation (11)

$$
\begin{aligned}
\mathbb{E} & {\left[\exp \left(\frac{i t D_{n+1}}{n}\right)\right] } \\
& =\sum_{k=0}^{n} \exp \left(\frac{i t k}{n}\right) \int_{-\infty}^{\infty}\left(\begin{array}{l}
n \\
k
\end{array}\right)[1-F(\theta-x)]^{k}[F(\theta-x)]^{n-k} F(d x) \\
& =\int_{-\infty}^{\infty} \sum_{k=0}^{n}\left(\begin{array}{l}
n \\
k
\end{array}\right) \exp \left(\frac{i t k}{n}\right)[1-F(\theta-x)]^{k}[F(\theta-x)]^{n-k} F(d x) \\
& =\int_{-\infty}^{\infty}\left[(1-F(\theta-x)) \exp \left(\frac{i t}{n}\right)+F(\theta-x)\right]^{n} F(d x) \\
& =\int_{-\infty}^{\infty}\left[(1-F(\theta-x))\left(1+\frac{i t}{n}+o\left(\frac{1}{n}\right)\right)+F(\theta-x)\right]^{n} F(d x) \\
& =\int_{-\infty}^{\infty}\left[1+\frac{i t}{n}(1-F(\theta-x))+o\left(\frac{1}{n}\right)\right]^{n} F(d x) \\
\rightarrow & \int_{-\infty}^{\infty} \exp (i t(1-F(\theta-x))) F(d x) \text { as } n \rightarrow \infty,
\end{aligned}
$$

where the limit follows from dominated convergence theorem because

$$
\begin{aligned}
& \left|(1-F(\theta-x)) \exp \left(\frac{i t}{n}\right)+F(\theta-x)\right|^{2} \\
& =[(1-F(\theta-x)) \cos (t / n)+F(\theta-x)]^{2}+(1-F(\theta-x))^{2} \sin ^{2}(t / n) \\
& =(1-F(\theta-x))^{2}+2 F(\theta-x)(1-F(\theta-x)) \cos (t / n)+(F(\theta-x))^{2} \\
& \leq 1 .
\end{aligned}
$$

This proves Theorem 1 .

\section{Pair correlation}

Now suppose there are $n+2$ vertices labeled $1,2, \ldots n, n+1, n+2$ with corresponding random variables $X_{1}, \ldots, X_{n+2}$. Define

$$
\begin{aligned}
& D_{n, 1}:=D_{n+2}(n+1)=\#\left\{j: 1 \leq j \leq n+2, j \neq n+1, X_{j}+X_{n+1}>\theta\right\} \\
& D_{n, 2}:=D_{n+2}(n+2)=\#\left\{j: 1 \leq j \leq n+2, j \neq n+2, X_{j}+X_{n+2}>\theta\right\} .
\end{aligned}
$$

We have, for $0 \leq k, l \leq n+1$,

$$
\mathbb{P}\left[D_{n, 1}=k, D_{n, 2}=l\right]=\mathbb{P}\left[D_{n, 1}=l, D_{n, 2}=k\right]
$$


For $k>l$

$$
\begin{aligned}
& \mathbb{P}\left[D_{n, 1}=k, D_{n, 2}=l\right] \\
& =\int_{-\infty}^{\infty} \int_{-\infty}^{\infty} 1_{\{a>b\}} F(d a) F(d b) \mathbb{P}\left[\#\left\{1 \leq j \leq n: a+X_{j}>\theta\right\}+1_{\{a+b>\theta\}}=k,\right. \\
& \left.\#\left\{1 \leq j \leq n: b+X_{j}>\theta\right\}+1_{\{a+b>\theta\}}=l\right] \\
& =\int_{-\infty}^{\infty} \int_{-\infty}^{\infty} 1_{\{a>b\}} 1_{\{a+b>\theta\}} F(d a) F(d b) \mathbb{P}\left[\#\left\{1 \leq j \leq n: a+X_{j}>\theta\right\}=k-1,\right. \\
& \left.\#\left\{1 \leq j \leq n: b+X_{j}>\theta\right\}=l-1\right] \\
& +\int_{-\infty}^{\infty} \int_{-\infty}^{\infty} 1_{\{a>b\}} 1_{\{a+b \leq \theta\}} F(d a) F(d b) \mathbb{P}\left[\#\left\{1 \leq j \leq n: a+X_{j}>\theta\right\}=k,\right. \\
& \left.\#\left\{1 \leq j \leq n: b+X_{j}>\theta\right\}=l\right] \\
& =\int_{-\infty}^{\infty} \int_{-\infty}^{\infty} 1_{\{a>b\}} 1_{\{a+b>\theta\}} g_{n}(\theta ; a, b ; k-1, l-1) F(d a) F(d b) \\
& +\int_{-\infty}^{\infty} \int_{-\infty}^{\infty} 1_{\{a>b\}} 1_{\{a+b \leq \theta\}} g_{n}(\theta ; a, b ; k, l) F(d a) F(d b),
\end{aligned}
$$

where, for $a \geq b$,

$$
\begin{aligned}
& g_{n}(\theta ; a, b ; k, l) \\
:= & \mathbb{P}\left[\#\left\{1 \leq j \leq n: a+X_{j}>\theta\right\}=k, \#\left\{1 \leq j \leq n: b+X_{j}>\theta\right\}=l\right] \\
= & \mathbb{P}\left[\#\left\{1 \leq j \leq n: X_{j}>\theta-a\right\}=k, \#\left\{1 \leq j \leq n: X_{j}>\theta-b\right\}=l\right] \\
= & \mathbb{P}\left[\#\left\{1 \leq j \leq n: X_{j}>\theta-b\right\}=l, \#\left\{1 \leq j \leq n: \theta-a<X_{j} \leq \theta-b\right\}=k-l\right] \\
= & \begin{cases}\frac{n !}{l !(k-l) !(n-k) !}[1-F(\theta-b)]^{l}[F(\theta-b)-F(\theta-a)]^{k-l}[F(\theta-a)]^{n-k} & \text { if } k \geq l \geq 0 \\
0 & \text { otherwise. }\end{cases}
\end{aligned}
$$

For $k=l$ similar calculations show

$$
\begin{aligned}
\mathbb{P}[ & \left.D_{n, 1}=k, D_{n, 2}=k\right] \\
= & \int_{-\infty}^{\infty} \int_{-\infty}^{\infty} 1_{\{a>b\}} 1_{\{a+b>\theta\}} g_{n}(\theta ; a, b ; k-1, k-1) F(d a) F(d b) \\
& +\int_{-\infty}^{\infty} \int_{-\infty}^{\infty} 1_{\{a>b\}} 1_{\{a+b \leq \theta\}} g_{n}(\theta ; a, b ; k, k) F(d a) F(d b) \\
& +\int_{-\infty}^{\infty} \int_{-\infty}^{\infty} 1_{\{a \leq b\}} 1_{\{a+b>\theta\}} g_{n}(\theta ; b, a ; k-1, k-1) F(d a) F(d b) \\
& +\int_{-\infty}^{\infty} \int_{-\infty}^{\infty} 1_{\{a \leq b\}} 1_{\{a+b \leq \theta\}} g_{n}(\theta ; b, a ; k, k) F(d a) F(d b) .
\end{aligned}
$$

Now let

$$
d_{n}(1):=\#\left\{j: 1 \leq j \leq n, X_{j}+X_{n+1}>\theta\right\}
$$


and

$$
d_{n}(2):=\#\left\{j: 1 \leq j \leq n, X_{j}+X_{n+2}>\theta\right\}
$$

then

$$
D_{n, 1} \geq d_{n}(1) \geq D_{n, 1}-1
$$

and

$$
D_{n, 2} \geq d_{n}(2) \geq D_{n, 2}-1
$$

Thus the asymptotic distributions of $\left(\frac{D_{n, 1}}{n}, \frac{D_{n, 2}}{n}\right)$ and $\left(\frac{d_{n}(1)}{n}, \frac{d_{n}(2)}{n}\right)$ are identical, and we work out pair correlation with $d_{n}(1)$ and $d_{n}(2)$ instead of $D_{n, 1}$ and $D_{n, 2}$.

Now, for all $0 \leq k, l \leq n$,

$$
\mathbb{P}\left[d_{n}(1)=k, d_{n}(2)=l\right]=\mathbb{P}\left[d_{n}(1)=l, d_{n}(2)=k\right],
$$

and, for $k>l$

$$
\mathbb{P}\left[d_{n}(1)=k, d_{n}(2)=l\right]=\int_{-\infty}^{\infty} \int_{-\infty}^{\infty} 1_{\{a>b\}} g_{n}(\theta ; a, b ; k, l) F(d a) F(d b),
$$

while, for $k=l$

$$
\begin{aligned}
\mathbb{P}\left[d_{n}(1)=k, d_{n}(2)=k\right]= & \int_{-\infty}^{\infty} \int_{-\infty}^{\infty} 1_{\{a>b\}} g_{n}(\theta ; a, b ; k, k) F(d a) F(d b) \\
& +\int_{-\infty}^{\infty} \int_{-\infty}^{\infty} 1_{\{a \leq b\}} g_{n}(\theta ; b, a ; k, k) F(d a) F(d b) \\
= & 2 \int_{-\infty}^{\infty} \int_{-\infty}^{\infty} 1_{\{a>b\}} g_{n}(\theta ; a, b ; k, k) F(d a) F(d b) \\
& +\int_{-\infty}^{\infty} \int_{-\infty}^{\infty} 1_{\{a=b\}} g_{n}(\theta ; a, b ; k, k) F(d a) F(d b) .
\end{aligned}
$$

Now let $-\infty<s, t<\infty$. To derive pair independence, let us consider the characteristic function. We have

$$
\mathbb{E}\left[\exp \left(i s d_{n}(1)+i t d_{n}(2)\right)\right]=\sum_{k=0}^{n} \sum_{l=0}^{n} \exp (i s k+i t l) \mathbb{P}\left[d_{n}(1)=k, d_{n}(2)=l\right]
$$

We break the above double sum into three parts, when (i) $k>l$, (ii) $k<l$ and (iii) $k=l$. 
For (i) we have

$$
\begin{aligned}
& \sum_{k, l: 0 \leq l<k \leq n} \exp (i s k+i t l) \mathbb{P}\left[d_{n}(1)=k, d_{n}(2)=l\right] \\
= & \sum_{k, l: 0 \leq l<k \leq n} \exp (i s k+i t l) \int_{-\infty}^{\infty} \int_{-\infty}^{\infty} 1_{\{a>b\}} g_{n}(\theta ; a, b ; k, l) F(d a) F(d b) \\
= & \int_{-\infty}^{\infty} \int_{-\infty}^{\infty} 1_{\{a>b\}} F(d a) F(d b) \sum_{k, l: 0 \leq l<k \leq n} \exp (i s k+i t l) g_{n}(\theta ; a, b ; k, l) .
\end{aligned}
$$

The term

$$
\begin{aligned}
& \sum_{k, l: 0 \leq l<k \leq n} \exp (i s k+i t l) g_{n}(\theta ; a, b ; k, l) \\
& =\sum_{k, l: 0 \leq l<k \leq n} \exp (i s k+i t l) \frac{n !}{l !(k-l) !(n-k) !} \\
& \times[1-F(\theta-b)]^{l}[F(\theta-b)-F(\theta-a)]^{k-l}[F(\theta-a)]^{n-k} \\
& =\sum_{k, l: 0 \leq l \leq k \leq n} \exp (i s(k-l)+i l(s+t)) \frac{n !}{l !(k-l) !(n-k) !} \\
& \times[1-F(\theta-b)]^{l}[F(\theta-b)-F(\theta-a)]^{k-l}[F(\theta-a)]^{n-k} \\
& -\sum_{k, l: 0 \leq l=k \leq n} \exp (i s(k-l)+i l(s+t)) \frac{n !}{l !(k-l) !(n-k) !} \\
& \times[1-F(\theta-b)]^{l}[F(\theta-b)-F(\theta-a)]^{k-l}[F(\theta-a)]^{n-k} \\
& =[\exp (i(s+t))(1-F(\theta-b))+\exp (i s)(F(\theta-b)-F(\theta-a))+F(\theta-a)]^{n} \\
& -\sum_{k=0}^{n} \exp (i k(s+t)) \frac{n !}{k !(n-k) !}[1-F(\theta-b)]^{k}[F(\theta-a)]^{n-k} \\
& =[\exp (i(s+t))(1-F(\theta-b))+\exp (i s)(F(\theta-b)-F(\theta-a))+F(\theta-a)]^{n} \\
& -[\exp (i(s+t))(1-F(\theta-b))+F(\theta-a)]^{n} \text {. }
\end{aligned}
$$


Rigorous results on the threshold network model

Thus

$$
\begin{aligned}
& \sum_{k, l: 0 \leq l<k \leq n} \exp (i s k+i t l) \mathbb{P}\left[d_{n}(1)=k, d_{n}(2)=l\right] \\
= & \int_{-\infty}^{\infty} \int_{-\infty}^{\infty} 1_{\{a>b\}} F(d a) F(d b) \\
& \left([\exp (i(s+t))(1-F(\theta-b))+\exp (i s)(F(\theta-b)-F(\theta-a))+F(\theta-a)]^{n}\right. \\
& \left.\quad-[\exp (i(s+t))(1-F(\theta-b))+F(\theta-a)]^{n}\right) .
\end{aligned}
$$

Similarly, for (ii),

$$
\begin{aligned}
& \sum_{k, l: 0 \leq k<l \leq n} \exp (i s k+i t l) \mathbb{P}\left[d_{n}(1)=k, d_{n}(2)=l\right] \\
= & \int_{-\infty}^{\infty} \int_{-\infty}^{\infty} 1_{\{a>b\}} F(d a) F(d b) \\
& \left([\exp (i(s+t))(1-F(\theta-b))+\exp (i t)(F(\theta-b)-F(\theta-a))+F(\theta-a)]^{n}\right. \\
& \left.\quad-[\exp (i(s+t))(1-F(\theta-b))+F(\theta-a)]^{n}\right) .
\end{aligned}
$$

For (iii),

$$
\begin{aligned}
& \sum_{k, l: 0 \leq k=l \leq n} \exp (i s k+i t k) \mathbb{P}\left[d_{n}(1)=k, d_{n}(2)=k\right] \\
= & 2 \int_{-\infty}^{\infty} \int_{-\infty}^{\infty} 1_{\{a>b\}} F(d a) F(d b) \\
& \left(\sum_{k=0}^{n} \exp (i s k+i t k) \frac{n !}{k !(n-k) !}(1-F(\theta-b))^{k}(F(\theta-a))^{n-k}\right) \\
& +\int_{-\infty}^{\infty} \int_{-\infty}^{\infty} 1_{\{a=b\}} F(d a) F(d b) \\
= & \int_{-\infty}^{\infty} \int_{-\infty}^{\infty} 1_{\{a>b\}} F(d a) F(d b)[(1-F(\theta-b)) \exp (i(s+t))+F(\theta-a)]^{n} \\
& \left.\left.+\int_{-\infty}^{\infty} \int_{-\infty}^{\infty} 1_{\{a=b\}} F(d a) F(d b)[(1-F(\theta-b)) \exp (i(s+t))+F(\theta-a)\}^{n}\right]^{n}\right)
\end{aligned}
$$


Combining the above and taking $s=u / n$ and $t=v / n$, we have

$$
\begin{aligned}
\mathbb{E} & {\left[\exp \left(i u\left(d_{n}(1) / n\right)+i v\left(d_{n}(2) / n\right)\right)\right] } \\
= & \int_{-\infty}^{\infty} \int_{-\infty}^{\infty} 1_{\{a>b\}} F(d a) F(d b) \\
& \left\{\left[\exp \left(\frac{i(u+v)}{n}\right)(1-F(\theta-b))+\exp \left(\frac{i u}{n}\right)(F(\theta-b)-F(\theta-a))+F(\theta-a)\right]^{n}(38)\right. \\
& \left.+\left[\exp \left(\frac{i(u+v)}{n}\right)(1-F(\theta-b))+\exp \left(\frac{i v}{n}\right)(F(\theta-b)-F(\theta-a))+F(\theta-a)\right]^{n}\right\} \\
& +\int_{-\infty}^{\infty} \int_{-\infty}^{\infty} 1_{\{a=b\}} F(d a) F(d b)\left[\exp \left(\frac{i(u+v)}{n}\right)(1-F(\theta-b))+F(\theta-a)\right]^{n} \\
= & \int_{-\infty}^{\infty} \int_{-\infty}^{\infty} 1_{\{a>b\}} F(d a) F(d b)\left\{\left[\left(1+\frac{i(u+v)}{n}+\frac{O(1)}{n^{2}}\right)(1-F(\theta-b))\right.\right. \\
& +\left[\left(1+\frac{i(u+v)}{n}+\frac{O(1)}{n^{2}}\right)(1-F(\theta-b))\right. \\
& \left.\left.+\left(1+\frac{i v}{n}+\frac{O(1)}{n^{2}}\right)(F(\theta-b)-F(\theta-a))+F(\theta-a)\right]^{n}\right\} \\
& \left.\left.+\int_{-\infty}^{\infty} \int_{-\infty}^{\infty} 1_{\{a=b\}} F(d a) F(d b)\left[\left(1+\frac{i(u+v)}{n}+\frac{O(1)}{n^{2}}\right)(1-F(\theta-b))+F(\theta-a)\right]^{n}\right]^{n}\right)
\end{aligned}
$$

Letting $n \rightarrow \infty$, we see that

$$
\begin{aligned}
& \lim _{n \rightarrow \infty} \mathbb{E}\left[\exp \left(i u\left(d_{n}(1) / n\right)+i v\left(d_{n}(2) / n\right)\right)\right] \\
= & \int_{-\infty}^{\infty} \int_{-\infty}^{\infty} 1_{\{a>b\}} F(d a) F(d b)\{\exp [i(u+v)(1-F(\theta-b))+i u(F(\theta-b)-F(\theta-a))] \\
& +\exp [i(u+v)(1-F(\theta-b))+i v(F(\theta-b)-F(\theta-a))]\} \\
& +\int_{-\infty}^{\infty} \int_{-\infty}^{\infty} 1_{\{a=b\}} F(d a) F(d b) \exp [i(u+v)(1-F(\theta-b))] \\
= & \int_{-\infty}^{\infty} \exp (i v(1-F(\theta-b))) F(d b) \int_{-\infty}^{\infty} \exp (i u(1-F(\theta-a))) F(d a) .
\end{aligned}
$$

Thus combining with the result in Section 2 we see that

$$
\begin{aligned}
& \lim _{n \rightarrow \infty} \mathbb{E}\left[\exp \left(i u\left(d_{n}(1) / n\right)+i v\left(d_{n}(2) / n\right)\right)\right] \\
& =\mathbb{E}\left[\exp \left(i u\left(1-F\left(\theta-X_{1}\right)\right)\right)\right] \mathbb{E}\left[\exp \left(i v\left(1-F\left(\theta-X_{1}\right)\right)\right)\right] \\
& =\lim _{n \rightarrow \infty} \mathbb{E}\left[\exp \left(i u\left(D_{n}(1) / n\right)\right)\right] \lim _{n \rightarrow \infty} \mathbb{E}\left[\exp \left(i v\left(D_{n}(2) / n\right)\right)\right],
\end{aligned}
$$

i.e. we obtain the asymptotic independence as enunciated in Theorem 2 ,

Now we obtain Theorem 3. The joint conditional probability distribution of the weights of two fixed vertices, provided that these vertices are connected 
by an edge, is given by

$$
H(d a, d b)=\frac{1(a+b>\theta)}{\alpha_{F}(\theta)} F(d a) F(d b)
$$

where $\alpha_{F}(\theta):=\int_{-\infty}^{\infty} \int_{-\infty}^{\infty} 1(\tilde{a}+\tilde{b}>\theta) F(d \tilde{a}) F(d \tilde{b})$ is the normalizing constant. It is actually the probability that two vertices share an edge. Consequently, the conditional probability distribution of weight of a vertex provided that it shares an edge with another vertex is given by

$$
\begin{aligned}
G(d a) & =\int_{-\infty}^{\infty} H(d a, d b) \\
& =F(d a) \frac{\int_{-\infty}^{\infty} 1(a+b>\theta) F(d b)}{\alpha_{F}(\theta)} \\
& =\frac{1-F(\theta-a)}{\alpha_{F}(\theta)} F(d a) .
\end{aligned}
$$

In order to study the asymptotic dependence of $d_{n}(1) / n$ and $d_{n}(2) / n$, we need to consider the difference between the expressions in the left and right sides of equation (40). Using equations (42) and (43), the asymptotic limit of this difference is

$$
\begin{aligned}
& \lim _{n \rightarrow \infty} \mathbb{E}\left[\exp \left(i u\left(d_{n}(1) / n\right)+i v\left(d_{n}(2) / n\right)\right)\right] \\
& -\lim _{n \rightarrow \infty} \mathbb{E}\left[\exp \left(i u\left(D_{n}(1) / n\right)\right)\right] \lim _{n \rightarrow \infty} \mathbb{E}\left[\exp \left(i v\left(D_{n}(2) / n\right)\right)\right] \\
= & \int_{-\infty}^{\infty}(H(d a, d b)-G(d a) G(d b)) e^{i u(1-F(\theta-a))} e^{i v(1-F(\theta-b))} .
\end{aligned}
$$

If the resulting conditional random variables are asymptotically independent, then for all $u, v \in \mathbb{R}$, the right hand side of equation (44) must vanish. Now we claim that this will imply that the probability measures $H(d a, d b)$ and $G(d a) G(d b)$ on $\mathbb{R}^{2}$ are identical. Indeed, let $\left(M_{1}, M_{2}\right)$ and $\left(R_{1}, R_{2}\right)$ be two random vectors on $\mathbb{R}^{2}$ whose distributions are given by the probability measures $H(d a, d b)$ and $G(d a) G(d b)$, respectively. Consider the map $\tilde{f}$ from $\mathbb{R}^{2} \rightarrow \mathbb{R}^{2}$ defined by $\tilde{f}:(a, b) \rightarrow(1-F(\theta-a), 1-F(\theta-b))$. Then, the supposition that equation (44) vanishes implies that the characteristic function of $\tilde{f}\left(M_{1}, M_{2}\right)$ is same as that of $\tilde{f}\left(R_{1}, R_{2}\right)$. Hence their distributions are also same. Therefore,

$$
\begin{aligned}
\mathbb{P} & \left.M_{1} \leq \beta_{1}, M_{2} \leq \beta_{2}\right] \\
& =\mathbb{P}\left[1-F\left(\theta-M_{1}\right) \leq 1-F\left(\theta-\beta_{1}\right), 1-F\left(\theta-M_{2}\right) \leq 1-F\left(\theta-\beta_{2}\right)\right] \\
& =\mathbb{P}\left[1-F\left(\theta-R_{1}\right) \leq 1-F\left(\theta-\beta_{1}\right), 1-F\left(\theta-R_{2}\right) \leq 1-F\left(\theta-\beta_{2}\right)\right] \\
& =\mathbb{P}\left[R_{1} \leq \beta_{1}, R_{2} \leq \beta_{2}\right] ;
\end{aligned}
$$


i.e. $\quad H(d a, d b)$ and $G(d a) G(d b)$ are identical.

Now, we claim that, because of Assumption 1 (of Section 1), the probability measures $H(d a, d b)$ and $G(d a) G(d b)$ cannot be the same. Indeed, if the measures $H(d a, d b)$ and $G(d a) G(d b)$ are same, then for any subset $A \subseteq \mathbb{R}^{2}$, $\int_{A} H(d a, d b)=\int_{A} G(d a) G(d b)$. Using the equations (42) and (43), we have

$$
\int_{A}\left[\frac{1(a+b>\theta)}{\alpha_{F}(\theta)}-\frac{1-F(\theta-a)}{\alpha_{F}(\theta)} \frac{1-F(\theta-b)}{\alpha_{F}(\theta)}\right] F(d a) F(d b)=0 .
$$

This will imply that

$$
1(a+b>\theta) \alpha_{F}(\theta)-(1-F(\theta-a))(1-F(\theta-b))=0
$$

$F \times F$ almost surely.

Now fix $u$ and $v$ as in Assumption 1 and let $\epsilon$ be such that $u+v-4 \epsilon>\theta$ and $u+4 \epsilon<\theta / 2<v-4 \epsilon$. Let $a, b \in(u-\epsilon, u+\epsilon]$. Then

$$
1(a+b>\theta) \alpha_{F}(\theta)=0 .
$$

However, $\theta-a<\theta-u+\epsilon<v-3 \epsilon<v-\epsilon$, so $1-F(\theta-a) \geq 1-F(v-\epsilon)$ $\geq F(v+\epsilon)-F(v-\epsilon)>0$. Similarly, $1-F(\theta-b)>0$. Thus

$$
(1-F(\theta-a))(1-F(\theta-b))>0 .
$$

Hence, on a set of probability at least $(F(v+\epsilon)-F(v-\epsilon))^{2}>0$ we have

$$
1(a+b>\theta) \alpha_{F}(\theta)-(1-F(\theta-a))(1-F(\theta-b))<0 .
$$

This contradiction completes the proof.

\section{Triangles}

Now we study the number of triangles in the graph. The number of triangles $T_{n}$ can be represented as a U-statistic (see [11]). The kernel function $h: \mathbb{R}^{3} \rightarrow \mathbb{R}$ defined in equation (4) is clearly a symmetric function of $x_{1}, x_{2}, x_{3}$. Then, we have

$$
T_{n}=\left(\begin{array}{l}
n \\
3
\end{array}\right) \times \frac{1}{\left(\begin{array}{l}
n \\
3
\end{array}\right)} \sum_{(i, j, k) \in C} h\left(X_{i}, X_{j}, X_{k}\right)=\left(\begin{array}{l}
n \\
3
\end{array}\right) U_{n}
$$

where $C:=\{(i, j, k): 1 \leq i<j<k \leq n\}$ is the collection of all possible triplets and $U_{n}:=\frac{1}{\left(\begin{array}{c}n \\ 3\end{array}\right)} \sum_{(i, j, k) \in C} h\left(X_{i}, X_{j}, X_{k}\right)$ is the U-statistic obtained from 
the kernel $h$. Theorem 4(a) can be easily derived from Theorem A of [1] (1980), page 190 .

Next define $h_{1}: \mathbb{R} \rightarrow \mathbb{R}$ as follows:

$$
\begin{aligned}
h_{1}(x) & :=\mathbb{E}\left[h\left(x, X_{2}, X_{3}\right)\right] \\
& =\mathbb{P}\left[X_{2}>\theta-x, X_{3}>\theta-x, X_{2}+X_{3}>\theta\right] \\
& =\mathbb{P}\left[\min \left\{X_{2}, X_{3}\right\}>\theta-x, X_{2}+X_{3}>\theta\right] .
\end{aligned}
$$

Noting that

$$
\mathbb{E}\left[h_{1}\left(X_{1}\right)\right]=\mathbb{E}\left[\mathbb{E}\left[h\left(X_{1}, X_{2}, X_{3}\right) \mid X_{1}\right]\right]=\mathbb{E}\left[h\left(X_{1}, X_{2}, X_{3}\right)\right]=F_{3}(\theta),
$$

we have

$$
\zeta_{1}(F)=\mathbb{V a r}\left(h_{1}(X)\right)>0
$$

unless $F$ is degenerate. The asymptotic normality of $T_{n} /\left(\begin{array}{l}n \\ 3\end{array}\right)$, i.e. Theorem 4 (b) follows from Theorem A of [11] (1980), page 192.

As an aside, we note that the method of U-statistics employed above is more versatile. It may be applied to configurations involving any subgraph composed of finitely many vertices.

Let $\mathbb{G}$ be a graph on $k$ vertices $(k \leq n)$ and suppose $\mathbb{V}:=\left\{v_{1}, \ldots, v_{k}\right\}$ and $\mathbb{E}$ be the vertex and edge sets of the graph, respectively. For a permutation $\sigma: \mathbb{V} \rightarrow \mathbb{V}$, let us consider the graph $\mathbb{G}_{\sigma}$ with vertex set $\mathbb{V}$ and edge set $\mathbb{E}_{\sigma}:=\left\{\left\langle\sigma\left(v_{i}\right), \sigma\left(v_{j}\right)\right\rangle:\left\langle v_{i}, v_{j}\right\rangle \in \mathbb{E}\right\}$. Let $l:=\#\left\{\sigma: \mathbb{E}_{\sigma}=\mathbb{E}\right\}$. Thus $l$ counts the number of symmetries of $\mathbb{G}$. To illustrate this, consider the graph with vertex set as $\mathbb{V}=\{1,2,3,4\}$ and edge set $\mathbb{E}=\{\langle 1,2\rangle,\langle 2,3\rangle,\langle 3,4\rangle,\langle 4,1\rangle\}$. For this graph $l=8$; indeed any of the eight permutations $(1,2,3,4),(2,3,4,1),(3,4,1,2),(4,1,2,3),(1,4,3,2),(4,3,2,1),(3,2,1,4)$ and $(2,1,4,3)$ result in a graph which is equivalent to the original graph.

Now given a graph $G$ on the vertex set $V:=\{1,2, \ldots, n\}$ with edge set $E$ consider the subgraph $G\left(i_{1}, \ldots, i_{k}\right)$ on the vertex set $V\left(i_{1}, \ldots, i_{k}\right)=\left\{i_{1}, \ldots, i_{k}\right\}$ and edge set $E\left(i_{1}, \ldots, i_{k}\right)=\left\{\left\langle i_{t}, i_{s}\right\rangle:\left\langle i_{t}, i_{s}\right\rangle \in E\right.$ and $\left.1 \leq t \neq s \leq k\right\}$. Let $\mathbb{L}_{k}$ be the set of all ordered $k$-tuples from $\{1,2, \ldots, n\}$ and $f: \mathbb{L}_{k} \rightarrow\{0,1\}$ be defined as follows:

$$
f\left(i_{1}, i_{2}, \ldots, i_{k}\right)= \begin{cases}1 & \text { if }\left\langle i_{s}, i_{t}\right\rangle \in E\left(i_{1}, i_{2}, \ldots, i_{k}\right) \text { for all }\left\langle v_{s}, v_{t}\right\rangle \in \mathbb{E} \\ 0 & \text { otherwise. }\end{cases}
$$

Thus, the number of subgraphs in $G$ isomorphic to $\mathbb{G}$ is given by $l^{-1} T_{n}(\mathbb{G})$, where

$$
T_{n}(\mathbb{G}):=\sum_{\left(i_{1}, i_{2}, \ldots, i_{k}\right) \in \mathbb{L}_{k}} f\left(i_{1}, i_{2}, \ldots, i_{k}\right)
$$


For the graph $G_{\theta}$, the vertices $i_{s}$ and $i_{t}$ are connected by an edge if and only if $X_{i_{s}}+X_{i_{t}}>\theta$. Thus the function $f$ in the definition (56) may be replaced by the kernel function $h: \mathbb{R}^{k} \rightarrow\{0,1\}$ defined by

$$
h\left(X_{i_{1}}, X_{i_{2}}, \ldots, X_{i_{k}}\right)= \begin{cases}1 & \text { if } X_{i_{s}}+X_{i_{t}}>\theta \text { for all }\left\langle v_{s}, v_{t}\right\rangle \in \mathbb{E} \\ 0 & \text { otherwise }\end{cases}
$$

and we have

$$
T_{n}(\mathbb{G})=\sum_{\left(i_{1}, i_{2}, \ldots, i_{k}\right) \in \mathbb{L}_{k}} h\left(X_{i_{1}}, X_{i_{2}}, \ldots, X_{i_{k}}\right) .
$$

This function $h$ need not be symmetric in its argument. Even if it were so, since the sum is over all ordered $k$-tuples $\left(i_{1}, \ldots, i_{k}\right)$ and not on $k$-tuples $\left(i_{1}, \ldots, i_{k}\right)$ such that $i_{1}<i_{2}<\cdots<i_{k}, T_{n}$ need not be a U-statistic.

To overcome these, we first define a symmetrized version of the kernel $h$. Let,

$$
h_{\mathrm{sym}}\left(x_{1}, x_{2}, \ldots, x_{k}\right):=\frac{1}{k !} \sum_{\tilde{\sigma} \in P(k)} h\left(x_{\tilde{\sigma}(1)}, x_{\tilde{\sigma}(2)}, \ldots, x_{\tilde{\sigma}(k)}\right)
$$

where $\tilde{\sigma}:\left\{x_{1}, x_{2}, \ldots, x_{k}\right\} \rightarrow\left\{x_{1}, x_{2}, \ldots, x_{k}\right\}$ is a permutation, and $P(k)$ is collection of all such permutations. We note that, even if $x_{1}=x_{2}$, we take $\left(x_{2}, x_{1}, x_{3}, \ldots, x_{k}\right)$ to be a permutation of $\left(x_{1}, x_{2}, x_{3}, \ldots, x_{k}\right)$. Clearly, $h_{\mathrm{sym}}$ is a symmetric function of $x_{1}, x_{2}, \ldots, x_{k}$, and we obtain

$$
T_{n}(\mathbb{G})=\sum_{\left(i_{1}, i_{2}, \ldots, i_{k}\right) \in \mathbb{L}_{k}} h\left(x_{i_{1}}, x_{i_{2}}, \ldots, x_{i_{k}}\right)=\sum_{\left(i_{1}, i_{2}, \ldots, i_{k}\right) \in \mathbb{L}_{k}} h_{\mathrm{sym}}\left(x_{i_{1}}, x_{i_{2}}, \ldots, x_{i_{k}}\right) .
$$

The statistic $T_{n}(\mathbb{G})$ is not a $U$-statistic. Thus we consider the statistic

$$
\begin{aligned}
T_{n}^{\prime}(\mathbb{G}) & =\sum_{i_{1}=1}^{n} \sum_{i_{2}=1}^{n} \ldots \sum_{i_{k}=1}^{n} h_{\text {sym }}\left(x_{i_{1}}, x_{i_{2}}, \ldots, x_{i_{k}}\right) \\
& =T_{n}+\sum_{\substack{1 \leq i_{1}, i_{2}, \ldots, i_{k} \leq n \\
\text { not all distinct }}} h_{\text {sym }}\left(x_{i_{1}}, x_{i_{2}}, \ldots, x_{i_{k}}\right) \\
& =T_{n}(\mathbb{G})+R_{n}(\mathbb{G}) \text { (say). }
\end{aligned}
$$

Since $T_{n}^{\prime}(\mathbb{G})$ is a von Mises' statistic (see [10], page 39), the asymptotic results about $T_{n}^{\prime}(\mathbb{G})$ can be read off from the results about the von Mises' statistics with the kernel function $h_{\text {sym }}$. To relate the statistics $T_{n}(\mathbb{G})$ and $T_{n}^{\prime}(\mathbb{G})$ we observe that the number of terms in the sum defining $R_{n}$ is of the order of $n^{k-1}$; thus noting that $h_{\text {sym }} \leq 1$, we have $R_{n}=O\left(n^{k-1}\right)$ as $n \rightarrow \infty$. Let

$$
F(\mathbb{G}):=\mathbb{E}\left[h\left(X_{1}, X_{2}, \ldots, X_{k}\right)\right] .
$$


Then, by the i.i.d. nature of $\left\{X_{i}: i \geq 1\right\}$, we have $\mathbb{E}\left[h_{\text {sym }}\left(X_{1}, X_{2}, \ldots, X_{k}\right)\right]=$ $F(\mathbb{G})$.

Theorem 8 As $n \rightarrow \infty$,

$$
\frac{T_{n}(\mathbb{G})}{n^{k}} \rightarrow F(\mathbb{G}) \text { almost surely. }
$$

Proof: From Theorem 3.3.1 of [10], page 102, we have $\frac{T_{n}^{\prime}(\mathbb{G})}{n^{k}} \rightarrow F(\mathbb{G})$ almost surely. Our observation that $R_{n}=O\left(n^{k-1}\right)$ as $n \rightarrow \infty$ completes the proof of the theorem.

To obtain the central limit theorem, as in (52) let

$$
h_{1}(x):=\mathbb{E}\left[h_{\mathrm{sym}}\left(x, X_{2}, X_{3}, \ldots, X_{k}\right)\right]
$$

and $\zeta_{1}(\mathbb{G})=\operatorname{Var}\left(h_{1}(X)\right)$ where $X$ is an independent random variable identical in distribution to $X_{1}$. Then, from Theorem 4.2.5 ([10], page 134) we have the central limit theorem for $T_{n}^{\prime}(\mathbb{G})$. Now $R_{n}(\mathbb{G}) / n^{k} \rightarrow 0$ in probability as $n \rightarrow \infty$. Thus we obtain

Theorem 9 As $n \rightarrow \infty$,

$$
\sqrt{n}\left[\frac{T_{n}(\mathbb{G})}{n^{k}}-F(\mathbb{G})\right] \Rightarrow \sqrt{k \zeta_{1}(\mathbb{G})} Z .
$$

\section{Local Properties}

In this section we study $T_{n}(1)$ as defined in equation (8).

For fixed $x \in \mathbb{R}$, the kernel $h\left(x, x_{1}, x_{2}\right)$ as defined in equation (4) is a symmetric function of $x_{1}$ and $x_{2}$. Define a U-statistic based on the kernel $h(x, \cdot, \cdot)$ by

$$
T_{n}(1 ; x):=\sum_{2 \leq i \neq j \leq n+1} h\left(x, X_{i}, X_{j}\right) .
$$

We have by the strong law for U-statistics (Theorem A, [11] page 190)

$$
\frac{T_{n}(1 ; x)}{\left(\begin{array}{l}
n \\
2
\end{array}\right)} \rightarrow \mathbb{E}\left[h\left(x, X_{i}, X_{j}\right)\right] \quad \text { almost surely, as } n \rightarrow \infty .
$$

The random variable $T_{n}(1 ; x)$ may be easily identified as the number of triangles of $G_{\theta}$ with a fixed vertex 1 and $X_{1}=x$. Formally, we may write, for any $t \in \mathbb{R}$

$$
\begin{aligned}
\mathbb{E}\left[\exp \left(i t \frac{T_{n}(1)}{\left(\begin{array}{l}
n \\
2
\end{array}\right)}\right)\right] & =\int_{\mathbb{R}} F(d x) \mathbb{E}\left[\exp \left(i t \frac{T_{n}(1)}{\left(\begin{array}{c}
n \\
2
\end{array}\right)}\right) \mid X_{1}=x\right] \\
& \rightarrow \int_{\mathbb{R}} F(d x) \exp \left(i t \mathbb{E}\left[h\left(x, X_{2}, X_{3}\right)\right]\right)
\end{aligned}
$$


as $n \rightarrow \infty$, because $T_{n}(1 ; x) /\left(\begin{array}{c}n \\ 2\end{array}\right) \rightarrow \mathbb{E}\left[h\left(x, X_{2}, X_{3}\right)\right]$ almost surely and hence also in distribution. The limit is justified by the usual application of dominated convergence theorem since the integrand, being a characteristic function, is bounded by 1 . The right hand side of (68) is the characteristic function of the random variable $\int_{\mathbb{R}} \int_{\mathbb{R}} F\left(d x_{1}\right) F\left(d x_{2}\right) h\left(X, x_{1}, x_{2}\right)$. This proves the Theorem 5 .

As in the previous section we may generalize Theorem 5 for the subgraph $\mathbb{G}$ defined earlier.

\section{Spatial model}

Consider the Poisson spatial model as elaborated in Section 1. We first thin the underlying Poisson process. Fix $x \in \mathbb{R}$. For $i \geq 1$, each point $\xi_{i}$ of the original process is included in the thinned process with a probability $f\left(\left|\xi_{i}\right| ; x\right)$ independently of the other points. The thinned process is an inhomogeneous Poisson process with intensity function $g(y)$ for $y \in \mathbb{R}$ given by $g(y)=\lambda f(|y| ; x)$, where $f(r ; x)$ is as defined in equation (111). For $\Delta_{r}$ as defined in equation (10), we have

Proposition 1 The conditional distribution of $\Delta_{r}$ given that $X_{0}=x$, is Poisson with parameter $\lambda_{r}(x)$ where

$$
\lambda_{r}(x):=\lambda \int_{|\tilde{r}| \leq r} f(\tilde{r} ; x) d \tilde{r} .
$$

We first prove Theorem [6] where $C_{r}(x) \rightarrow C(x)<\infty$ (see Section 1 for the relevant definitions). Because $t \rightarrow \int_{\mathbb{R}} F(d x) \exp \left(-\lambda c_{d} C(x)(1-\exp (i t))\right)$ is indeed a characteristic function, it is enough to prove that the characteristic function of $\Delta_{r}$ converges to the above quantity. By Proposition 1 the conditional distribution of $\Delta_{r}$ given $X_{0}=x$ is Poisson with parameter $\int_{|\tilde{r}| \leq r} \lambda f(\tilde{r}, x) d \tilde{r}=\lambda c_{d} \int_{0}^{r} \tilde{r}^{d-1}\left[1-F\left(\theta \tilde{r}^{\beta}-x\right)\right] d \tilde{r}=\lambda c_{d} C_{r}(x)$ where $c_{d}$ is the volume of the $(d-1)$-dimensional unit sphere. Therefore, we have

$$
\begin{aligned}
\phi_{\Delta_{r}}(t) & :=\mathbb{E}\left[\exp \left(i t \Delta_{r}\right)\right] \\
& =\int_{\mathbb{R}} F(d x) \mathbb{E}\left[\exp \left(i t \Delta_{r}\right) \mid X_{0}=x\right] \\
& =\int_{\mathbb{R}} F(d x) \exp \left(-\lambda c_{d} C_{r}(x)(1-\exp (i t))\right) .
\end{aligned}
$$

Now, since $C_{r}(x) \rightarrow C(x)$, the usual dominated convergence theorem assures

$$
\phi_{\Delta_{r}}(t) \rightarrow \phi_{\Delta}(t) \text { as } r \rightarrow \infty
$$


Rigorous results on the threshold network model

This completes the proof of Theorem 6.

Remark Assuming $\theta>0$, we may re-write $C(x)$ in the following way:

$$
\begin{aligned}
& \int_{0}^{\infty} r^{d-1}\left[1-F\left(\theta r^{\beta}-x\right)\right] d r \\
& =\int_{0}^{\infty} r^{d-1} \int_{-\infty}^{\infty} 1\left(\tilde{x}>\theta r^{\beta}-x\right) F(d \tilde{x}) d r \\
& =\int_{-\infty}^{\infty} \int_{0}^{\infty} r^{d-1} 1\left(r<\max \left\{0,[(\tilde{x}+x) / \theta]^{1 / \beta}\right\}\right) d r F(d \tilde{x}) \\
& =\frac{1}{d} \int_{-\infty}^{\infty}\left[\max \left\{0,[(\tilde{x}+x) / \theta]^{d / \beta}\right\}\right] F(d \tilde{x}) \\
& =\frac{1}{d \theta^{d / \beta}} \int_{-\infty}^{\infty}\left[\max \left\{0,(\tilde{x}+x)^{d / \beta}\right\}\right] F(d \tilde{x}) \\
& =\frac{1}{d \theta^{d / \beta}} \mathbb{E}\left[\max \left\{0,\left(X_{0}+x\right)^{d / \beta}\right\}\right]
\end{aligned}
$$

Thus $C(x)<\infty$ if $\mathbb{E}\left[\left|X_{0}\right|^{d / \beta}\right]<\infty$.

To show Theorem [7] it is enough to prove that the characteristic function of $\left(\Delta_{r}-\lambda c_{d} C_{r}\right) / \sqrt{\lambda c_{d} C_{r}}$ converges to the product of the characteristic functions of a standard normal random variable and $\sqrt{\lambda c_{d}} g\left(X_{0}\right)$.

$$
\begin{aligned}
& \mathbb{E}\left[\exp \left(i t \frac{\Delta_{r}-\lambda c_{d} C_{r}}{\sqrt{\lambda c_{d} C_{r}}}\right)\right] \\
= & \int_{\mathbb{R}} F(d x) \mathbb{E}\left[\exp \left(i t \frac{\Delta_{r}-\lambda c_{d} C_{r}}{\sqrt{\lambda c_{d} C_{r}}}\right) \mid X_{0}=x\right] \\
= & \int_{\mathbb{R}} F(d x) \exp \left(-i t \sqrt{\lambda c_{d} C_{r}}\right) \mathbb{E}\left[\exp \left(i \frac{t}{\sqrt{\lambda c_{d} C_{r}}} \Delta_{r}\right) \mid X_{0}=x\right] \\
= & \int_{\mathbb{R}} F(d x) \exp \left(-i t \sqrt{\lambda c_{d} C_{r}}-\lambda c_{d} C_{r}(x)\left(1-\exp \left(i t / \sqrt{\lambda c_{d} C_{r}}\right)\right)\right)
\end{aligned}
$$

using the fact that the conditional distribution of $\Delta_{r}$ given $X_{0}=x$ follows a Poisson distribution with parameter $\lambda c_{d} C_{r}(x)$. Consider the logarithm of the integrand:

$$
\begin{aligned}
& -i t \sqrt{\lambda c_{d} C_{r}}-\lambda c_{d} C_{r}(x)\left[1-\exp \left(\frac{i t}{\sqrt{\lambda c_{d} C_{r}}}\right)\right] \\
& =-i t \sqrt{\lambda c_{d} C_{r}}-\lambda c_{d} C_{r}(x)\left[-\frac{i t}{\sqrt{\lambda c_{d} C_{r}}}-\frac{1}{2}\left(\frac{i t}{\sqrt{\lambda c_{d} C_{r}}}\right)^{2}+o\left(\left(\frac{i t}{\sqrt{\lambda c_{d} C_{r}}}\right)^{2}\right)\right] \\
& =i t \sqrt{\lambda c_{d}}\left[\frac{C_{r}(x)-C_{r}}{\sqrt{C_{r}}}\right]-\frac{t^{2}}{2} \frac{C_{n}(x)}{C_{r}}-\lambda c_{d} C_{r}(x) \times o\left(\frac{1}{C_{r}}\right) .
\end{aligned}
$$


Under our condition, the first term converges to $i t \sqrt{\lambda c_{d}} g(x)$. The condition also implies that $C_{r}(x) / C_{r} \rightarrow 1$ as $r \rightarrow \infty$, and thus the second term converges to $-t^{2} / 2$. The third term can be written as $\left[C_{r}(x) / C_{r}\right] \times\left[C_{r} O\left(1 / C_{r}\right)\right] \rightarrow 0$ as $r \rightarrow \infty$. Applying the dominated convergence theorem, we now obtain that

$$
\mathbb{E}\left[\exp \left(i t \frac{\Delta_{r}-\lambda c_{d} C_{r}}{\sqrt{\lambda c_{d} C_{r}}}\right)\right] \rightarrow \exp \left(-\frac{t^{2}}{2}\right) \int_{\mathbb{R}} F(d x) \exp \left(i t \sqrt{\lambda c_{d}} g(x)\right)
$$

Now note that $\exp \left(-t^{2} / 2\right)$ is the characteristic function of a standard normal random variable and $\int_{\mathbb{R}} F(d x) \exp \left(i t \sqrt{\lambda c_{d}} g(x)\right)$ is the characteristic function of $\sqrt{\lambda c_{d}} g\left(X_{0}\right)$. This completes the proof of Theorem 7

\section{Acknowledgments}

We thank Hiroyoshi Miwa for helpful discussion. Naoki Masuda is supported by Special Postdoctoral Researchers Program of RIKEN, and Rahul Roy is supported by DST Grant MS/152/01.

[1] Albert R Barabási A.-L. 2002 Rev. Mod. Phys. 7447.

[2] Newman M E J 2003 SIAM Rev. 45167.

[3] Caldarelli G Capocci A De Los Rios P Muñoz M A 2002 Phys. Rev. Lett. 89258702.

[4] Söderberg B 2002 Phys. Rev. E 66066121.

[5] Boguña M and Pastor-Satorras R 2003 Phys. Rev. E 68036112.

[6] Masuda N Miwa H and Konno N 2004 Phys. Rev. E 70036124.

[7] Masuda N Miwa H and Konno N 2005 Phys. Rev. E 71036108.

[8] Itzkovitz S Milo R Kashtan N Ziv G and Alon U 2003 Phys. Rev. E 68026127.

[9] Milo R Shen-Orr S Itzkovitz S Kashtan N Chklovskii D and Alon U 2002 Science 298 824.

[10] Koroljuk V S and Borovskich Yu V 1994 Theory of U-Statistics, (Dordrecht: Kluwer).

[11] Serfling R J 1980 Approximation Theorems of Mathematical Statistics, (New York: Wiley). 